\title{
Measurements of Quadrupole Magnets for the MIT-Bates SHR*
}

\author{
J.D. Zumbro, P. Bonneau, M. Farkhondeh, J.B. Flanz, \\ S.P. Holmberg, T. Russ, W.W. Sapp, and C. Sibley \\ Massachusetts Institute of Technology \\ Bates Linear Accelerator Center \\ Middleton, MA 01949.
}

\begin{abstract}
Results of mensurements on 24 of the 128 new quadrupole magnets for the pulse storage/stretcher ring currently under construction at the MIT-Bates Accelerator Center are presented.
\end{abstract}

\section{Introduction}

The MIT-Bates Linear Accelerator Center has an electron linac with a maximum energy of $\sim 500 \mathrm{MeV}$, but with the capability of putting the beam through the accelerator twice, a maximum electron energy of $1 \mathrm{GeV}$ can be achieved. The maximum duty factor of the accelerator is $\sim 1 \% ; 600-\sim 16 \mu$ sec pulses per second. The South Hall Ring (SHR) under construction is a pulse storage ring with $190 \mathrm{~m}$ circumference. This ring will allow $100 \%$ duty factor for internal target experiments and with two-turn injection circulating currents as large as $80 \mathrm{~mA}$ should be available. Simulations indicate duty factors in excess of $85 \%$ should be available for extracted beams. This project is described in greater detail elsewhere. [1]

For the SHR project 128 new quadrupoles magnets have been purchased. The accelcrator physicists have specified the initial setability of the quadrupoles is to be $0.1 \%$ and limits of the harmonic content such that $\Sigma\left|B_{n} / B_{2}\right| \leq$ 0.02 at $30 \mathrm{~mm}$. While this may seem like a large harmonic content, note that this is at $92.3 \%$ of full aperture for these quadrupoles.

The quadrupole magnets are laminated with the nominal length of the iron being $280 \mathrm{~mm}$. The pole aperture is nominally $65 \mathrm{~mm}$ diameter and the pole-tip shape of these quadrupoles is the same as that for the booster-ring quadrupole magnets for the Advanced Light Source currently under construction at the Lawrence-Berkley Laboratories. The steel, purchased from Inland Steel [2], had a carbon content of $\leq 0.008 \%$ (by weight). The laminations for the Bates SHR quadrupoles were stamped by Electrometal Products [3] using the same die as used for the LBL quadrupole laminations. Everson Electric [4] made the coils and assembled these magnets.

- Wirk suppurted in part by DOE cuntract DE $\Lambda$ C02-76ER03069.

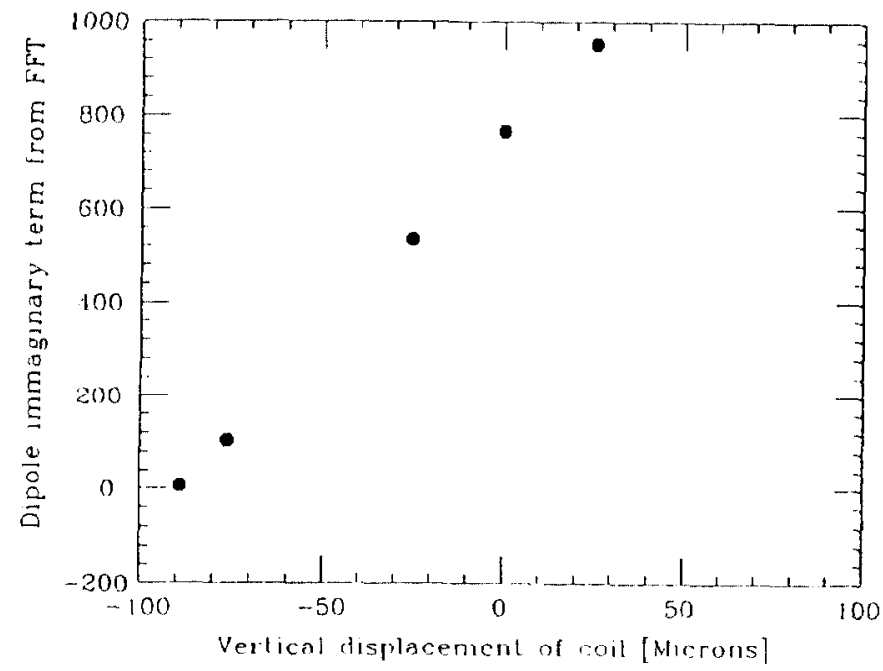

Figure 1: Sensitivity of coil position to the dipole component of a quadrupole.

\section{Measurements}

At the outset it was decided to measure excitation curves for every quadrupole, and at the same time have enough sensitivity to extract the harmonic content. We are using a measuring machine built for us at Chalk River.[5] Our machine is a slightly larger version of one that they used for measuring quadrupole magnets for an upgrade at their TASCC facility. Our machine has two separate tangential coils with nominal opening angles of $90^{\circ}$ and $45^{\circ}$ wonnd of a ceramic $\left(\mathrm{Al}_{2} \mathrm{O}_{3}\right)$ bobbin. These coils have 47 and $4 \mathrm{R}$ turns with mean radii of 28.56 and $28.75 \mathrm{~mm}$, respectively, and are $800 \mathrm{~mm}$ long. While the coil assembly is being rotated with a frequency of $\sim 1 / 2$ Hertz, pulses from a $V / f$ converter whose input is from one of the coils are accumulated into 256 angle bins per revolution - this data acquisition is controlled by an AST-286 PC. During production measurements we obtain 5 scans in the clockwise direction and 5 scans in the counter-clockwise direction at each quadrupole current. The $\mathrm{PC}$ also has a BITBUS card used for power supply control and a GPIB card that is used to read the magnet current via a voltmeter and current transformer. During production measurements the computer 
rearls the voltage that is proportional to the current after each forward/reverse pair, this number is recorded in the same file as the data from the rotating coil. A Fast-Fourier Transform (FFT) is later performed on the data and with the correct geometry for the coil the quantity $\left(\mathrm{B}_{0} \mathrm{~L}_{e j f} / \mathrm{a}\right)$ is extracted. $\mathrm{L}_{e f f}$ is the effective length of the quadrupole and is $\sim 300 \mathrm{~mm}$ as determine by other measurement, and $a$ is the aperture radius, $32.5 \mathrm{~mm}$.

The following table shows the raw FFT amplitudes for four different quadrupoles when energized to about the same integrated strength. Two of these quads are SHR quadrupoles ( $32.5 \mathrm{~mm}$ aperture) and the other two are 38 $\mathrm{mm}$-aperture quadrupoles that were removed from a beam line that was dismantled at the start of construction on the ring. The purpose of this table is to emphasize the sensitivity of the machine to higher harmonics, if they are present. This data was obtained using the $90^{\circ}$ coil and the *'s indicates terms that this coil is not sensitive to. Quad CQ3B was found, subsequent to its measurement, to have one coil with $3 \%$ greater voltage drop.

\begin{tabular}{|c|c|c|c|c|}
\hline \multirow[b]{2}{*}{$\mathbf{n}$} & \multicolumn{4}{|c|}{ Rav FFT amplitudes } \\
\hline & CQ3A & CQ3B & SHR Q019 & SHR Q062 \\
\hline $\mathbf{0}$ & 0.1766 & 0.0344 & 0.0531 & $0.1563 *$ \\
\hline 1 & 0.9660 & 0.4175 & 1.2108 & 2.8494 \\
\hline 2 & 5140.6590 & 5199.2870 & 5167.1600 & 5179.8207 \\
\hline 3 & 6.5647 & 44.9232 & 5.6501 & 3.2225 \\
\hline 4 & 1.7882 & 1.7759 & 1.4870 & $0.8287 *$ \\
\hline 5 & 7.5050 & 2.7483 & 1.7522 & 1.4038 \\
\hline 6 & 34.3748 & 40.1028 & 4.5050 & 4.8830 \\
\hline 7 & 0.6046 & 2.5063 & 0.5398 & 0.3886 \\
\hline 8 & 0.4650 & 0.5296 & 0.8598 & $0.7785 *$ \\
\hline 9 & 0.8792 & 2.2992 & 0.1756 & 0.5493 \\
\hline 10 & 25.1223 & 26.0819 & 1.5227 & 1.1420 \\
\hline 11 & 0.4326 & 1.4721 & 0.4530 & 0.5821 \\
\hline 12 & 0.1476 & 0.1317 & 0.2972 & $0.1294 *$ \\
\hline 13 & 0.2326 & 0.3473 & 0.3981 & 0.4295 \\
\hline 14 & 1.3065 & 1.8072 & 0.3796 & 0.3365 \\
\hline 15 & 0.3250 & 0.3182 & 0.4328 & 0.3575 \\
\hline
\end{tabular}

Before the measurements begin the bobbin axis is centered in the quadrupole in two distinct steps. First, a mechanical alignment spindle is substituted for the bobbin and the pitch and yawl adjusted by using the five digital-readout micrometers ( $1 \mu \mathrm{m}$ resolution) which are an integral part of the bobbin/spindle support structure -3 for the vertical motion and 2 for the horizontal motion. For these quadrupoles the mechanical centering precision is limited by the asymmetry of the bore aperture. While this asymmetry has not resulted in unacceptable harmonic content, it has required us to use a survey fiducialization scheme which is not referenced to the mechanical axes. This scheme is described elsewhere in these proceedings. After the initial alignment step is completed and the bobbin is reinstalled if the magnet has never been energized at Bates it is ramped between 0 and 150 amps and back to 0 ten times. After ramping we complete the centering procedure by energixing the magnet to $50 \mathrm{nmps}(\sim 2.7$ kG pole-tip field) - at this point the micrometers are used to adjust the coil position to zero (actually minimize) the dipole component observed in FFT analysis of a scan in the quadrupole. This procedure is considered complete when micrometer changes are less than $\sim 10 \mu \mathrm{m}$. Figure 1 shows the sensitively of the vertical displacement of the coil to a quadrupole's dipole component.

In production measurements after the coil is aligned the $\mathrm{V} / \mathrm{f}$ converter input is shorted and a voltage source is substituted. The output of a frequency meter counting the $\mathrm{V} / \mathrm{f}$ converter output and a voltmeter that is also hookedto the voltage source is record as the voltage source is step from positive full scale range of the $V / f$ converter to ininus full scale range. This calibration process is computer controlled and these instruments are read/controlled using a GPIB card in the PC.

\section{Results}

The advertised operating range of the SHR is $300 \mathrm{MeV}$ to $1 \mathrm{GeV}$, this and the optics specify that the range of quadrupole currents in the SHR will be from $\sim 5$ to $\sim 150$ amps. This along with the management's desire to keep the cost of the project low by having quadrupoles of the same type or family in the injection line, the ring lattice, and the extraction line energized in series, and the accelerator physicist's goal of initial setup of the quadrupoles to $0.1 \%$, require that data be taken for several different maximum cycling currents. We are in fact taking data at five different maximum currents $(150,100,60,50$, and 40 amps) - the most probable power supply sizes. Figure 2 show a typical excitation curve for one quadrupole when cycled to 150 amps. Figure 3 show a comparison of data for Q058 when compared to value predicted from parameters obtained from fitting the $150 \mathrm{amp}$ cycle data of Q062. The functional form used to fit the data is

$$
\begin{aligned}
B_{0} L_{e f f} / a(I)= & a_{0}+a_{1}(I / 150)+a_{3}(I / 150)^{3}+\cdots \\
& +a_{13}(I / 150)^{13}
\end{aligned}
$$

where $B_{i 1} L_{c f J} / a$ is determined from data from the harmonic analyser and I is the current at whirh the mrasure ment was made. The deviations of the data for varions different cycling currents in Figure 3 indicate that it is clearly important as to how a magnet is cycled.

In our cycling procedures we ramp at the rate of 2 amps/sec and remain at the maximum current for $10 \mathrm{sec}-$ onds before starting to ramp down to current of interest, again at the rate of $2 \mathrm{amps} / \mathrm{sec}$. Measurements indicate that we can re-measure a quadrupoles strength and reproduce the value of $\left(B_{0} L_{E J J} / a\right)$ versus current to better than $0.1 \%$. We have at this time not investigated how important the ramp rate is and the length of time that we wait at the top current. For these quadrupoles, observation of 


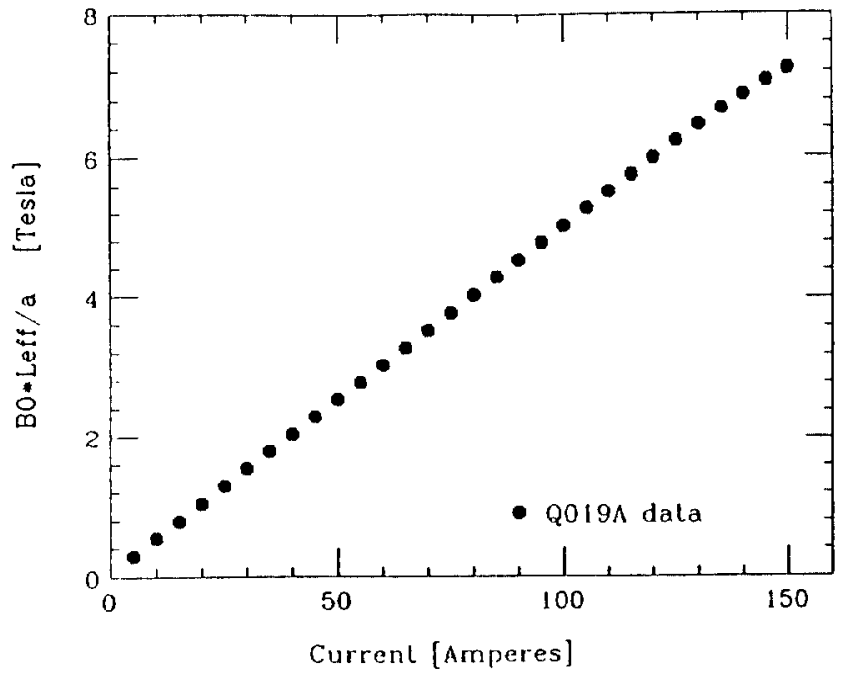

Figure 2: The quantity $\left(\mathrm{B}_{0} \mathrm{~L}_{e / f} / \mathrm{a}\right)$ versus current for a SHR quadrupole. $L_{e f f}$ is $\sim 300 \mathrm{~mm}$ and $\mathrm{a}$ is nominally $32.5 \mathrm{~mm}$.

the signal from the coil when it is in the quadrupole indicates that the field is not changing immediately after the quadrupole has stopped ramping. Obviously if these were not laminated magnets we would have to wait for a longer period of time.

Measurements also indicate that we can reverse the polarity of a quadrupoles remnant field by repeated cycling between 0 and 150 amps, and obtain the same results (to the $0.1 \%$ level) as was obtained previously. Possibly this is because we initially cycle the magnet between 0 and 150 amps 10 times prior to the first measurement. Figure 4 shows the values of $\mathrm{a} 0$ that we obtain from fitting the 150 amp cycle data to Eq. 1 - this term is due to the remnant field of the magnet. Figure 5 shows the value of the linear term (a 1 in Eq. 1) for the 24 quadrupoles measured thus far.

Measurements continue and we studying the data looking for quadrupoles that match. We periodically remeasure Q062, which has been defined to be the reference quadrupole, to check the consistency of the measurements.

\section{References}

[1] J.B. Flanz et al., Proceedings of the 1989 IEEE Particle Accelerator Conference, March 20-23, 1989, p.34.

[2] Inland Steel Company, Chicago, IL.

[3] Electro-metal Products Corp., Skokie, IL.

[4] Everson Electric Company, Lehigh Valley, PA.

[5] W.G. Davies, N. Bray, and R. Howard, Chalk River Nuclear Laboratories, Chalk River, Ontario, Canada; and W.G. Davies, submited to Nuclear Instruments and Methods for an analysis of the theory of tangential rotating coil measurements.

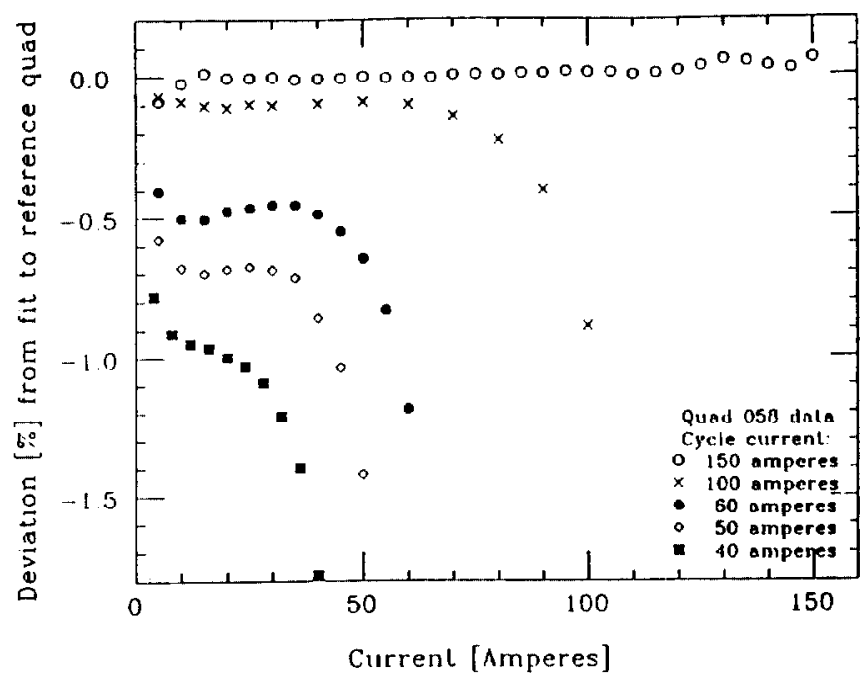

Figure 3: Comparision of measurements on one quadrupole (Q058) to the parameters determined by fitting Eq. 1 to data from the reference quadrupole (Q062).

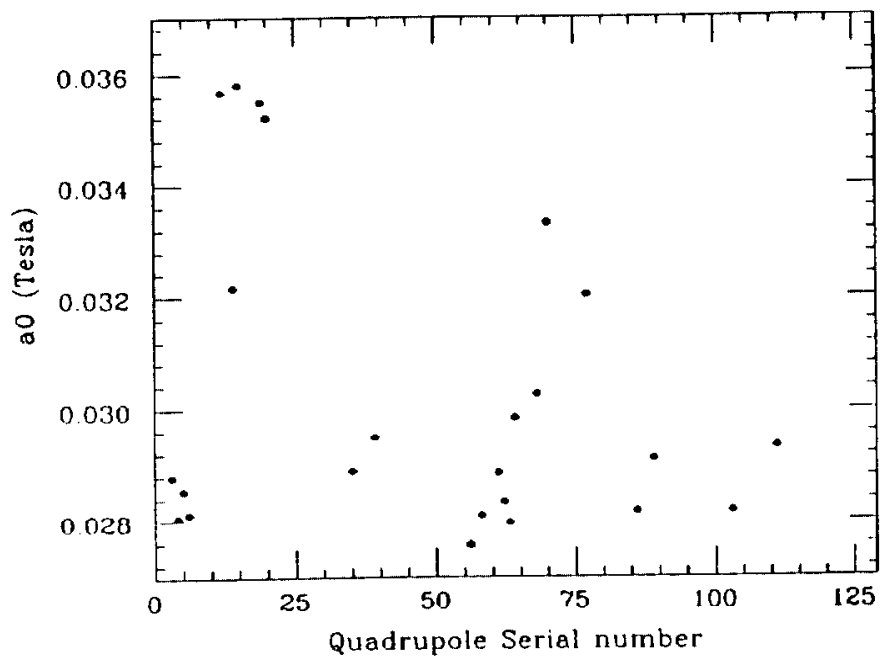

Figure 4: a0 from fits of 150 amp cycle data to Eq. 1 for quadrupoles measured to date.

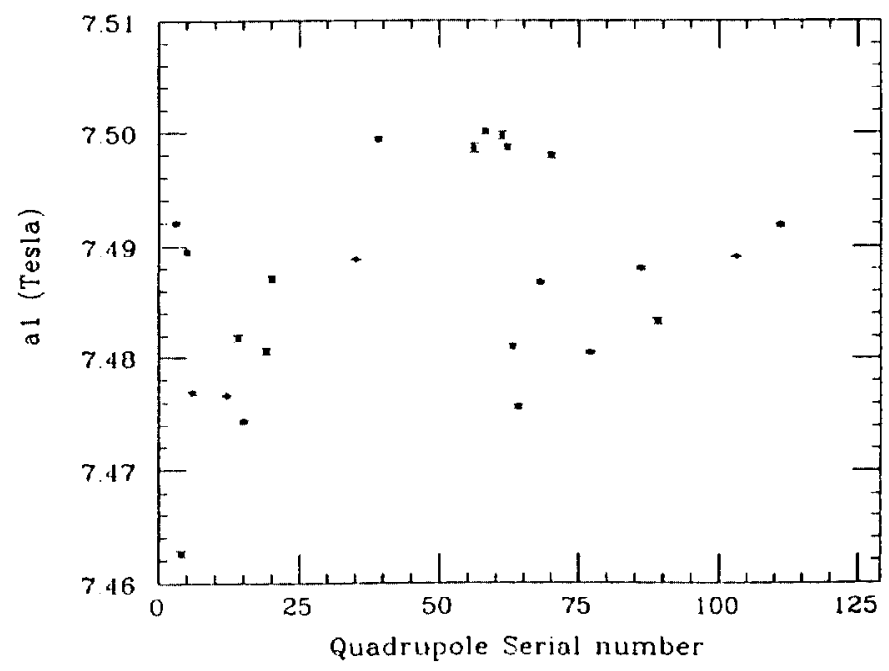

Figure 5: a1 from fits of 150 amp cycle data to Eq. 1 for quadrupoles measured to date. 